\title{
Flemish rivers phytoplankton
}

Maxime Sweetlove, Jeroen Van Wichelen, Elie Verleyen \& Wim Vyverman
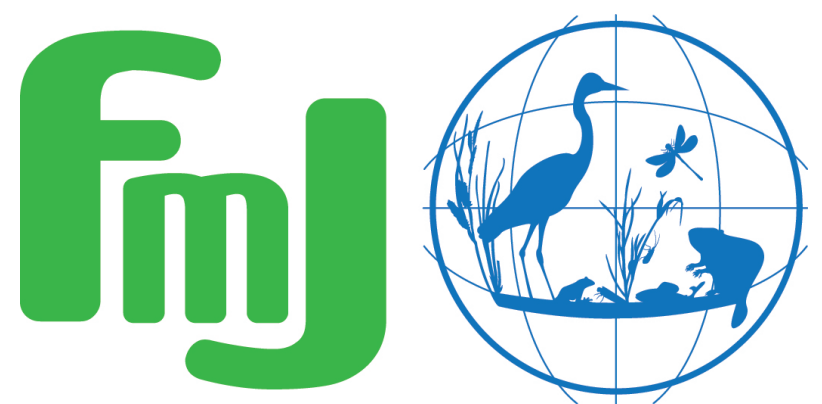

Freshwater Metadata Journal 



\title{
Flemish rivers phytoplankton
}

\author{
Maxime Sweetlove ${ }^{1,3}$, Jeroen Van Wichelen ${ }^{1,2}$, Elie Verleyen ${ }^{1} \&$ Wim Vyverman ${ }^{1}$ \\ 1 Ghent University, Ghent, Belgium \\ 2 Instituut voor stuur en Bosonderzoek, Brussels, Belgium \\ 3 Royal Belgian Institute for Natural Sciences, Brussels, Belgium
}

Please cite this paper as follows: Sweetlove, M., Van Wichelen, J., Verleyen, E. \& Vyverman, W., 2018. Flemish rivers phytoplankton. Freshwater Metadata Journal 39: 1-4.

https://doi.org/10.15504/fmj.2018.39

Received: 2018-10-16 / Published: 2018-10-18

\section{Keywords}

phytoplankton, rivers, channels, Flanders

\section{Short description of the dataset/summary}

Monitoring data from Flemish rivers and channels. The data contains phytoplankton counts of Flemish rivers and channels.

\section{General information}

dataset entry ID:

name of the dataset:

full name of the dataset:

dataset short name:

type of dataset:

data type:
SF_19

Flemish rivers phytoplankton

Flemish rivers

species distribution data

point data/observation data

science keywords according to GCMD:

topic:

Biosphere, Biological Classification, Terrestrial Hydrosphere

ISO topic category according to ISO 19115:

Biota, Environment, Inland Waters

INSPIRE keywords according to GEMET:

Environmental monitoring facilities, Species distribution

own science keywords:

funding:

phytoplankton, rivers, Flanders

INBO, VMM 


\section{Technical and administrative specifications}

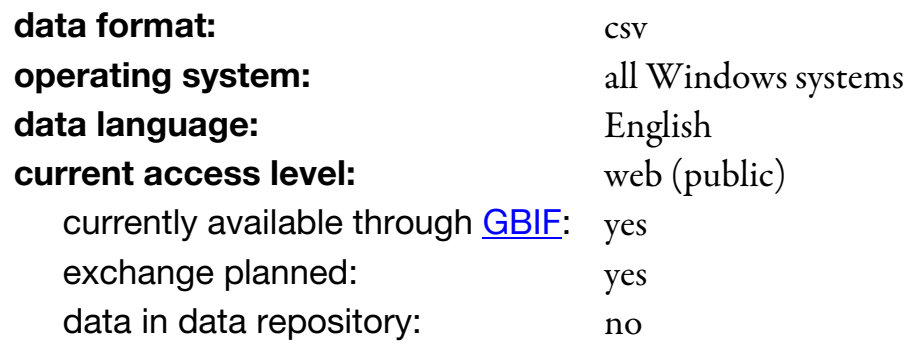

Do you plan to publish the data on the Freshwater Biodiversity Data Portal:

$\begin{array}{ll}\begin{array}{l}\text { update level: } \\ \text { documentation: } \\ \text { type: }\end{array} & \text { completed } \\ \text { language: } & \text { internal description } \\ & \text { Dutch } \\ \text { contact details: } & \\ \text { metadata contact person: } & \\ \text { first, last name: } & \text { Jeroen Van Wichelen } \\ \text { phone: } & +32025250277 \\ \text { email: } & \text { jeroen.vanwichelen@inbo.be } \\ \text { institution: } & \text { Instituut natuur- en bosonderzoek (INBO) } \\ \text { address: } & \text { Kliniekstraat } 25 \\ \text { postal code, city: } & 1070 \text { Anderlecht } \\ \text { country } & \text { Belgium } \\ \text { technical contact person: } & \\ \text { first, last name: } & \text { Jeroen Van Wichelen } \\ \text { phone: } & +32025250277 \\ \text { email: } & \text { jeroen.vanwichelen@inbo.be } \\ \text { scientific contact person: } & \\ \text { first, last name: } & \text { Jeroen Van Wichelen } \\ \text { phone: } & +32025250277 \\ \text { email: } & \text { jeroen.vanwichelen@inbo.be }\end{array}$

\section{Intellectual property rights and citation}

dataset creator (data compiler):

contact name:

Wim Vyverman

contact email:

wim.vyverman@ugent.be

contact institution:

Ghent University

data contributors to/owners of this dataset:

multiple

number:

2

\section{data contributor/owner 1:}

contact name:

Wim Vyverman

contact email:

wim.vyverman@ugent.be

contact institute:

Ghent University

criteria for using this part of the dataset:

The dataset is publicly available (data portal, data archive) and can be used without restrictions, but dataset creator/data contributors must be informed 
data contributor/owner 2:

contact name:

contact email:

contact institute:

criteria for using this part of the dataset:

The dataset is publicly available (data portal, data archive) and can be used without restrictions, but dataset creator/data contributors must be informed prior to publication. Data must be acknowledged and cited correctly.

citation of this dataset:

author(s):

Van Wichelen, J., Fagot, M., Lavens, F. \& Vyverman, W.

title and journal (name, number, pages):

Fytoplanktonanalyses van een aantal Vlaamse waterlopen ten behoeve van de Europese Kaderrichtlijn Water (VMM.ARW.001.2008)

year:

2008

doi:

citation of the metadata:

author(s):

https://doi.org/10.15468/e2181a

title and journal (name, number, pages):

Flemish rivers phytoplankton. Freshwater Metadata Journal 39: 1-4

year:

2018

doi:

\section{General data specifications}

regional coverage of the dataset:

spatial extent of the dataset:

continents:

regional

countries:

Europe

Europe: Belgium

world climatic regions according to Köppen:

European ecoregions according to Illies (WFD):

ecosystem type:

covered timeframe:

\section{Site specifications}

coordinate system/grid data:

grid data available:

number of sites:

\section{Biological data}

\section{biological data origin:}

organism group addressed: latitude/longitude, format: DD

no

$<100$
Western Plains (ER13)

rivers

$2007-2007$ from sampling,

Vlaamse Mileumaatschappij VMM.ARW.001.2008

phytoplankton 


\section{Sample resolution}

phytoplankton:

taxonomic resolution:

level: genus

taxonomic coding:

taxalist according to: no reference taxalist used

coding system: no coding

\section{Other specifications}

GIS layers, shape files related to the dataset:

no data available

availability of photos:

no

availability of maps:

no

quality control procedures:

Were any quality control procedures applied to your dataset?

no

\section{Acknowledgements}

The authors thank the Belgian Science Policy (Belspo) for funding the SAFRED project (Saving freshwater biodiversity research data) that allowed processing and publishing these data. 